\title{
Correction to: Microbial pigments as an alternative to synthetic dyes and food additives: a brief review of recent studies
}

\author{
Masoud Aman Mohammadi ${ }^{1} \cdot$ Hossein Ahangari $^{2}$ (1) $\cdot$ Saeed Mousazadeh ${ }^{2} \cdot$ Seyede Marzieh Hosseini $^{1}$. \\ Laurent Dufossé ${ }^{3}$
}

Published online: 15 October 2021

๑) Springer-Verlag GmbH Germany, part of Springer Nature 2021

\section{Correction to: Bioprocess and Biosystems Engineering https://doi.org/10.1007/s00449-021-02621-8}

The Acknowledgements section should be modified as

This study is related to the project no. 1398/9812 from Student Research Committee, Shahid Beheshti University of Medical Sciences, Tehran, Iran. We also appreciate the "Student Research Committee" and "Research \& Technology Chancellor" in Shahid Beheshti University of Medical Sciences for their financial support of this work. Laurent Dufossé deeply thanks the Conseil Régional de La Réunion, Indian Ocean, for continuous financial support of research projects dedicated to microbial pigments and shows gratitude to Mireille Fouillaud and Yanis Caro for many years of close association in microbial pigments research.

The Original Article was corrected.

Publisher's Note Springer Nature remains neutral with regard to jurisdictional claims in published maps and institutional affiliations.
The original article can be found online at https://doi.org/10.1007/ s00449-021-02621-8.

Seyede Marzieh Hosseini sm_hosseini@sbmu.ac.ir

$\triangle$ Laurent Dufossé

laurent.dufosse@univ-reunion.fr

1 Student Research Committee, Department of Food Science and Technology, National Nutrition and Food Technology Research Institute, Faculty of Nutrition Sciences, Food Science and Technology, Shahid Beheshti University of Medical Sciences, Tehran, Iran

2 Department of Food Science and Technology, Faculty of Nutrition and Food Sciences, Tabriz University of Medical Sciences, Tabriz, Iran

3 CHEMBIOPRO Lab, Ecole Supérieure d'Ingénieurs Réunion Océan Indien (ESIROI), Université de La Réunion, Département Agroalimentaire, 97744 Saint-Denis, France 